\title{
An SPQR-Tree Approach to Decide Special Cases of Simultaneous Embedding with Fixed Edges
}

\author{
J. Joseph Fowler ${ }^{1}$, Carsten Gutwenger ${ }^{2}$, Michael Jünger ${ }^{3, \star}$, \\ Petra Mutzel$^{2}$, and Michael Schulz ${ }^{3, \star}$ \\ ${ }^{1}$ Department of Computer Science, University of Arizona, USA \\ jfowler@cs.arizona.edu \\ ${ }^{2}$ Department of Computer Science, Technische Universität Dortmund, Germany \\ \{petra.mutzel, carsten.gutwenger\}@cs.tu-dortmund. de \\ ${ }^{3}$ Department of Computer Science, University of Cologne, Germany \\ \{mjuenger, schulz\}@informatik.uni-koeln.de
}

\begin{abstract}
We present a linear-time algorithm for solving the simultaneous embedding problem with fixed edges (SEFE) for a planar graph and a pseudoforest (a graph with at most one cycle) by reducing it to the following embedding problem: Given a planar graph $G$, a cycle $C$ of $G$, and a partitioning of the remaining vertices of $G$, does there exist a planar embedding in which the induced subgraph on each vertex partite of $G \backslash C$ is contained entirely inside or outside $C$ ? For the latter problem, we present an algorithm that is based on SPQR-trees and has linear running time. We also show how we can employ SPQR-trees to decide SEFE for two planar graphs where one graph has at most two cycles and the intersection is a pseudoforest in linear time. These results give rise to our hope that our SPQR-tree approach might eventually lead to a polynomial-time algorithm for deciding the general SEFE problem for two planar graphs.
\end{abstract}

\section{Introduction}

Many practical graph drawing applications demand planar embeddings of a graph that yield additional constraints. One natural application is in obtaining simultaneous drawings of a set of related planar graphs. This is useful in the areas of bioinformatics, social sciences and software engineering. A single drawing can be insufficient in depicting complex interrelationships of different models of a system. Instead, multiple drawings may be required, each from a different perspective. The challenge is to preserve the "mental map" of the common structures in each layout so that the scientist can easily navigate between the different diagrams. To do this, common vertices and edges are placed and drawn equally in each drawing. This can be modeled via embedding constraints.

Various embedding constraints have already been studied in [25|6]; Gutwenger et al. [12] apply SPQR-trees to efficiently decide if a graph has a combinatorial embedding with respect to a set of hierarchical constraints modeling grouping and fixed orders of edges around a vertex. We instead address a problem that cannot be modeled by any

\footnotetext{
^ Partially supported by the German Science Foundation (JU204/11-1).
} 
of the previous approaches. Given a planar graph $G$, a cycle $C \subset G$, and a partition $P$ of all vertices of $G \backslash C$, we ask whether there is a planar embedding of $G$ where all vertices $v \in p$ for some part $p \in P$ lie completely inside or outside $C$. We give an efficient decision algorithm using SPQR-trees that can be used to solve a simultaneous embedding problem.

Given a set of planar graphs $\left\{G_{1}, G_{2}, \ldots, G_{n}\right\}$ on the same vertex set, a simultaneous embedding with fixed edges (SEFE) of $\left\{G_{i}\right\}$ are planar drawings $\Gamma_{i}$ of $G_{i}$, $i \in[1 . . n]$, such that all vertices and all edges belonging to two graphs $G_{i}$ and $G_{j}$ are drawn identically in the corresponding drawings $\Gamma_{i}$ and $\Gamma_{j}$. SEFE and its variant of simultaneous geometric embedding (SGE) with planar straight-line drawings as well as the other variations of simultaneous embedding have become an important branch within the field of graph drawing. It is known that deciding SEFE is NP-complete for three graphs [11] while deciding SGE is NP-hard for two graphs [8]. The complexity of deciding SEFE for two graphs is still open.

Many approaches have been made to decide the problem for some classes of graph pairs [4|7|9]10]. Frati [10] showed that trees and planar graphs always have a SEFE. Fowler et al. [9] improved this result to show that forests, circular caterpillars (removal of all degree-1 vertices yields a cycle), $K_{4}$, and subgraphs of $K_{3}$-multiedges (an edge $(x, y)$ with any number of edges with $x$ or $y$ as endpoints) are the only graphs to always have a SEFE with any planar graph. Their drawing algorithms are based upon using an optimal Euclidean shortest path algorithm [13]. We also apply this technique in our algorithms.

In this paper we examine the pairs of a planar graph $G_{1}$ with a pseudoforest $G_{2}$. A SEFE is not always guaranteed unless all non-cycle edges of $G_{2}$ are incident to the cycle, i.e., the pseudoforest happens to be a circular caterpillar. However, we show that SEFE for such pairs can be decided in polynomial time by presenting an efficient decision algorithm. We further discuss efficient decision algorithms for the case that $G_{2}$ contains two cycles and $G_{1} \cap G_{2}$ is a pseudoforest. We think that our approach is promising in that it may eventually lead to a general polynomial time decision algorithm for testing SEFE of two graphs.

\section{Preliminaries}

Given some planar drawing $\Gamma$ of a planar graph $G$, a cycle $C$ in $G$ forms a Jordan curve that splits the plane into two connected components. One is bounded by $C$ and the other is unbounded as given by the Jordan curve theorem [14]. We say that some vertex $v \in G \backslash C$ lies in the interior (exterior) of $C$ if it is mapped to a position in the bounded (unbounded) component.

A combinatorial embedding of a planar graph $G$ is defined as a clockwise ordering of the incident edges for each vertex with respect to a crossing-free drawing of $G$ in the Euclidean plane. A planar embedding is a combinatorial embedding together with a fixed external face.

A block is a maximal 2-connected subgraph of a graph $G$. If $G$ is 2-connected, the $S P Q R$-tree $\mathcal{T}$ of $G$ represents its decomposition into 3-connected components comprising serial, parallel, and 3-connected structures [3]. The respective structure is given by a 
skeleton graph associated with each tree node which is either a cycle (S-node), a bundle of parallel edges (P-node), or a 3-connected simple graph (R-node); Q-nodes serve as representatives for the edges of $G$.

If $G$ is 2 -connected and planar, its SPQR-tree $\mathcal{T}$ represents all combinatorial embeddings of $G$. In particular, a combinatorial embedding of $G$ uniquely defines a combinatorial embedding of each skeleton in $\mathcal{T}$, and fixing the combinatorial embedding of each skeleton uniquely defines a combinatorial embedding of $G$.

Given two planar graphs $G_{1}=\left(V, E_{1}\right)$ and $G_{2}=\left(V, E_{2}\right)$ on the same vertex set $V$, a simultaneous embedding with fixed edges (SEFE) consists of planar drawings $\Gamma_{i}$ of $G_{i}, i \in[1,2]$, such that each vertex is mapped to the same point in the plane for $\Gamma_{1}$ and $\Gamma_{2}$ and each edge in $G_{1} \cap G_{2}$ is represented by the same simple curve in the plane for both drawings.

\section{A Planar Graph, a Cycle, and a Partition}

In this section, we consider the following graph embedding problem. Given a planar graph $G=(V, E)$, a cycle $C=\left(V_{C}, E_{C}\right) \subset G$, and a partition $P$ of $V \backslash V_{C}$, decide whether $G$ has a planar drawing such that all vertices of each part in $P$ either lie completely inside or outside of $C$; see Algorithm 1

The input partition $P$ or the planar embeddings of the graph may force two vertices to be on the same side of the cycle (either both inside or both outside). We call this situation a same-side constraint. On the other hand, by examining all embeddings of the graph we may reveal that two vertices must be positioned on opposite sides of the cycle (one inside and one outside). We refer to this situation as an opposite-side constraint. The idea of the algorithm is to find all such constraints and then check whether all these constraints can be satisfied at once, i.e., whether a planar embedding with the required property exists.

The following algorithm uses an SPQR-tree $\mathcal{T}$ to examine all embeddings of the block of graph $G$ containing the given cycle $C$. Each skeleton of a node of $\mathcal{T}$ may lead to constraints prohibiting some of the possible embeddings as discussed above. We use an auxiliary graph $H$ containing all of the vertices of the original graph to maintain the occuring constraints. Same-side constraints are represented by green edges and opposite-side constraints by red edges.

We say that $H$ is 2-colorable if its vertices can be colored with two colors, say red and green, in such a way that both endpoints of a green edge have the same color and both endpoints of a red edge have different color.

As cycles are 2-connected, the given cycle $C$ is contained in a single block $B$ of graph $G$. All other blocks are either completely inside or outside of $C$ in all planar drawings of $G$. Hence, we get one same-side constraint for all vertices of each block $B^{\prime} \neq B$. We can now assume to deal with a 2 -connected graph $G$ and its SPQR-tree $\mathcal{T}$ that represents all planar embeddings of $G$ together with some cycle $C \subseteq G$. Let $\nu \in T$ be some node of the SPQR-tree, $S$ be its skeleton and $e \in S$ be any skeleton edge. If the expansion graph of $e$ includes any edge of $C$, we call $e$ a cycle edge. We consider the different possibilities for $\nu$ in turn. 


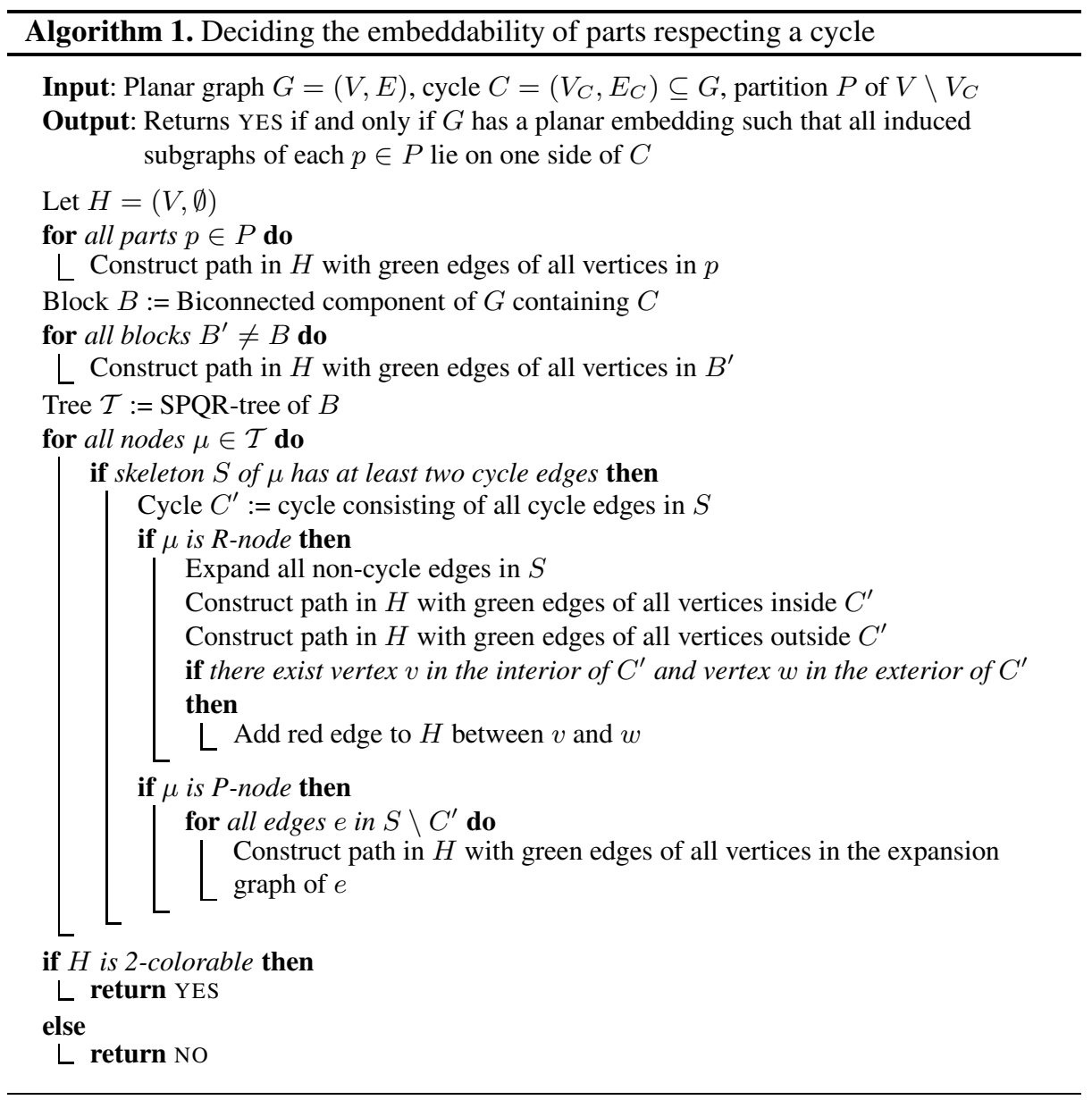

If $S$ contains exactly one cycle edge $e$, then the edges belonging to the skeleton of all the other vertices must lie on the same side of $C$. When regarding the node of $\mathcal{T}$ belonging to $e$, all these vertices are contained in the expansion graph of a single edge that is not a cycle edge. Repeating this process, if necessary, we get a $\mathcal{T}$-node that has more than one cycle edge but also has a single non-cycle edge containing all of the vertices from above. When dealing with this $\mathcal{T}$-node, the necessary auxiliary graph augmentation to handle this same-side constraint is performed.

If $S$ contains two or more cycle edges, then these cycle edges comprise a cycle in $S$. If $S$ also contains non-cycle edges, $\nu$ is a P-node or an R-node.

1. In an S-node this can only occur if all edges of the skeleton are cycle edges. In this situation there is nothing to be done as this does not lead to any same-side constraints or opposite-side constraints.

2. Let $\nu$ be a P-node (see Fig. 1). All the vertices occurring in an expansion graph of any other edge in $S$ are forced to be on one side of the cycle $C$. 


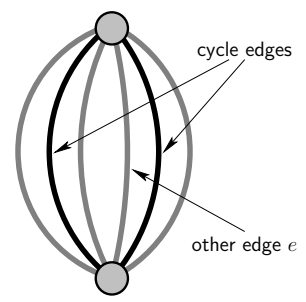

Fig. 1. Cycle edges in the skeleton of a P-node lead to same-side constraints: all vertices in the expansion graph of a non-cycle edge $e$ are on the same side of the cycle

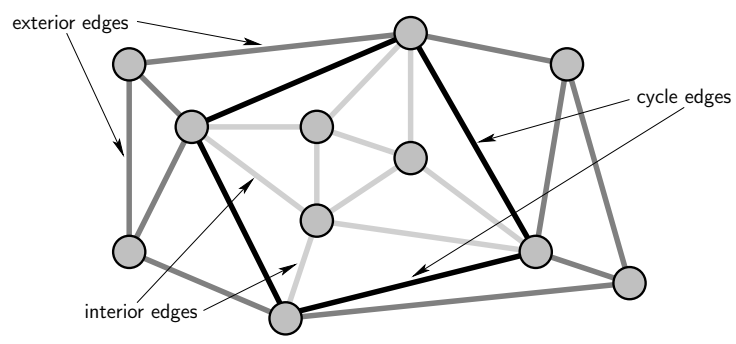

Fig. 2. Cycle edges in the skeleton of an R-node yield two same-side and one opposite-side constraints: All vertices in the expansion graphs of the interior component are on one side of the cycle while all vertices of the exterior component are on the other side

3. Having $\nu$ as an R-node (see Fig. 2) is the most involved. The skeleton $S$ of $\nu$ is a 3-connected graph and has hence a unique embedding (besides mirroring and choosing the outer face). The cycle edges split $S$ into two halves: the interior and the exterior components of $S$. All vertices belonging to all expansion graphs of edges of one side must be on one side of the cycle in the final embedding. Neither pair of vertices $w_{1}$ and $w_{2}$ being the interior and the exterior components, respectively, may end up on the same side of the cycle. Hence, we get two same-side constraints (between all vertices in the interior and exterior components, respectively) and one opposite-side constraint (the edges from the interior and the exterior components must be separated).

Theorem 1. Algorithm 1 has a runtime of $O(|V|)$ and works correctly, i.e., it returns YES if and only if the input graph $G$ has a planar embedding $\mathcal{E}$ such that for each $p \in P$ all vertices in plie on one side of $C$ in $\mathcal{E}$.

Proof. Obviously, the first two for-loops including the construction of $\mathcal{T}$ require only $O(|V|)$ time, thus add only $O(|V|)$ green edges to $H$. The third loop iterates over all nodes $\mu \in \mathcal{T}$ and expands some non-cycle edges. Observe that-for all nodes $\mu$ - the expansion graphs of these non-cycle edges do not share any edge, and thus no vertex except for vertices on the cycle $C$. Therefore, the whole for-loop takes $O(|V|)$ time, 
and we add only $O(|V|)$ green and red edges to $H$. Since the size of $H$ is linear in $|V|$, we can check if $H$ is 2-colorable using breadth-first-search in $O(|V|)$ time.

We next show that the algorithm works correctly. First, assume that the algorithm returns NO. Then the constructed auxiliary graph $H$ is not 2-colorable. This means that two vertices $v$ and $w$ in $H$ are connected by two paths: one containing an odd number of red edges and one containing an even number. This implies that $v$ and $w$ must lie on the same side of $C$ (due to the path with even number of red edges), as well as on opposite sides (path with odd number of red edges). Hence, $G$ has no such embedding.

Next assume that the algorithm returns YES in which $H$ is 2-colorable. We pick one of the two colors to lie in the interior of $C$ and one to lie on the outside. The choice of embeddings for every P-node and every R-node implies an embedding $\mathcal{E}$ for $G$. For each such node in $\mathcal{T}$, we can choose an embedding that satisfies the given choice of interior and exterior of $C$. In each P-node, the vertices that belong to the expansion graph of one of the parallel edges are connected by green edges in $H$, thus, they lie on the same side in $\mathcal{E}$. In each R-node, the vertices on both sides of the cycle are connected by green edges, respectively, while a single edge between these sets forbids both parts to lie on the same side. Finally, green edges between the vertices of the input partition yield that these vertices lie on the same side of $C$.

\section{A Planar Graph, a Pseudoforest, and a Decision}

In this section, we apply Algorithm 1 to solve the following open problem in simultaneous embedding: Given a planar graph $G_{1}$ and a pseudoforest $G_{2}$, find an efficient algorithm to decide whether the pair $\left\{G_{1}, G_{2}\right\}$ has an SEFE; see Algorithm 2, For a few special cases of $G_{2}$ the situation becomes trivial as described by the next theorem.

Theorem 2 (Fowler et al. [9]). Let $G_{1}$ be a planar graph and $G_{2}$ be a forest or a circular caterpillar. Then $G_{1}$ and $G_{2}$ have a SEFE.

Next, we consider the more general case of a pseudoforest containing a cycle $C$ in which not all non-cycle edges are incident to $C$. We see by the next theorem that the case is also trivial if $C$ is not in the intersection of $G_{1}$ and $G_{2}$.

Theorem 3. Let $G_{1}=\left(V, E_{1}\right)$ be a planar graph and $G_{2}=\left(V, E_{2}\right)$ be a pseudoforest with a cycle $C$. If $C$ is not in $G_{1} \cap G_{2}$, then the pair has a SEFE.

Proof. Let edge $e \in C \backslash G_{1}$. Create a planar drawing of $\Gamma_{1}$ of $G_{1}$ in the plane using any suitable graph drawing algorithm (e.g. [1]). We construct a planar drawing $\Gamma_{2}$ of $G_{2}$ that, together with $\Gamma_{1}$, creates a SEFE of $G_{1}$ and $G_{2}$.

Draw all vertices and all edges of $G_{1} \cap G_{2}$ in $\Gamma_{2}$ in the same way as in $\Gamma_{1}$ guaranteeing a simultaneous drawing. We still must draw all edges of $G_{2} \backslash G_{1}$ without introducing any crossings in $\Gamma_{2}$. As $e$ is not part of $G_{1}$, it has not been drawn in $\Gamma_{2}$ yet. We draw all edges of $G_{2} \backslash G_{1}$ in $\Gamma_{2}$ one after another with $e$ as the last edge. The order of the other edges can be chosen arbitrarily.

To do this we use an optimal Euclidean shortest path algorithm [13]. We apply the modification as done by Fowler et al. [9] in their drawing algorithms. A distance $\varepsilon$ is 
always maintained between the shortest path and any line segment corresponding to previous part of $\Gamma_{2}$. This allows subsequent edges to be routed as need be in between any pair of non-incident edges that would otherwise be touching. Applying this algorithm adds at most $O(|V|)$ edge bends for each new edge (as new bends hide old bends as argued in [9]) so that the final complexity of the drawing is $O\left(|V|^{2}\right)$ giving an overall running time of $O\left(|V|^{2} \log |V|\right)$.

As $G_{2}$ has only one cycle $C$ and $e$ is part of $C, G_{2} \backslash\{e\}$ is a forest. Any drawing of any subgraph of $G_{2} \backslash\{e\}$ has exactly one face. Hence, starting with the partial drawing of $\Gamma_{2}$ it is always possible to insert a route for the edges not yet drawn maintaining planarity. Even, in the last step, when edge $e$ is inserted, the partial drawing of $\Gamma_{2}$ has exactly one face and thus, $e$ can be safely inserted into $\Gamma_{2}$. Then $\Gamma_{2}$ is completed and $\left\{\Gamma_{1}, \Gamma_{2}\right\}$ is a SEFE of $\left\{G_{1}, G_{2}\right\}$.

Due to Theorems 2 and 3 we assume $G_{2}$ to have exactly one cycle $C$ in the intersection $G_{1} \cap G_{2}$. By construction $G_{1}$ is planar. However, to ensure a SEFE of $G_{1}$ and $G_{2}$ we must embed $G_{1}$ in such a way that the cycle $C$ does not separate any pair of vertices that are adjacent in $G_{2}$. On the other hand, as $G_{2} \backslash C$ is a forest, this condition suffices to guarantee a SEFE of the pair $\left\{G_{1}, G_{2}\right\}$.

Theorem 4. Let $G_{1}=\left(V, E_{1}\right)$ be a planar graph and $G_{2}=\left(V, E_{2}\right)$ be a pseudoforest each on $n$ vertices with a cycle $C \subseteq G_{1} \cap G_{2} . G_{1}$ and $G_{2}$ have $a$ SEFE if and only if there exists a planar drawing of $G_{1}$ such that for all edges $e=\{v, w\} \in G_{2} \backslash G_{1}$ either both $v$ and $w$ lie inside or both lie outside of $C$.

Proof. Assume first that $G_{1}$ has a planar drawing $\Gamma_{1}$ with the described property. We create a planar drawing $\Gamma_{2}$ of $G_{2}$ that, together with $\Gamma_{1}$, yields a SEFE of $G_{1}$ and $G_{2}$. Draw all vertices and all edges of $G_{1} \cap G_{2}$ in $\Gamma_{2}$ in the same way as in $\Gamma_{1}$. As $C \subseteq G_{1} \cap G_{2}$, the cycle is now present in $\Gamma_{2}$. We draw all remaining edges of $G_{2} \backslash G_{1}$ next by using the same approach of the proof of Theorem 3 .

We start with the edges $e$ that have one endpoint in the exterior of $C$ in $\Gamma_{1}$. Due to the condition on $\Gamma_{1}$, both endpoints of $e$ are in the exterior of $C$ or one endpoint is on $C$. As we have just drawn $C$ and all vertices in the same way as in $\Gamma_{1}$, this condition also holds for the partial drawing of $\Gamma_{2}$. As $G_{2} \backslash G_{1}$ is a forest there is a way to route $e$ without introducing crossings: Imagine $C$ and its interior as one big vertex. The partial drawing $\Gamma_{2}$ then has exactly one face. This also holds for edges connecting the exterior of $C$ with $C$ itself. The same argument holds for all the edges in the interior of $C$ as well as the edges connecting the interior with $C$. Hence, by construction we have a planar drawing $\Gamma_{2}$ of $G_{2}$ that, together with $\Gamma_{1}$, yields a SEFE of $G_{1}$ and $G_{2}$.

Now let $G_{1}$ be without a planar drawing with the described property. Assume $G_{1}$ and $G_{2}$ have a SEFE. By definition there exist planar drawings $\Gamma_{i}$ of $G_{i}, i \in[1,2]$, such that the intersection $G_{1} \cap G_{2}$ is drawn in the same way in both $\Gamma_{1}$ and $\Gamma_{2}$. As $G_{1}$ has no planar drawing with the described property, there exists an edge $e=\{v, w\} \in G_{2} \backslash C$ such that $v$ lies in the interior of $C$ and $w$ lies in the exterior of $C$ in $\Gamma_{1}$. As vertices $v$ and $w$ and cycle $C$ are part of $G_{1} \cap G_{2}$, the same condition holds for $\Gamma_{2}$. But this means that $e$ cannot be routed in $\Gamma_{2}$ without introducing a crossing in $\Gamma_{2}$, which is a contradiction to our assumption. Hence, $G_{1}$ and $G_{2}$ have no SEFE. 


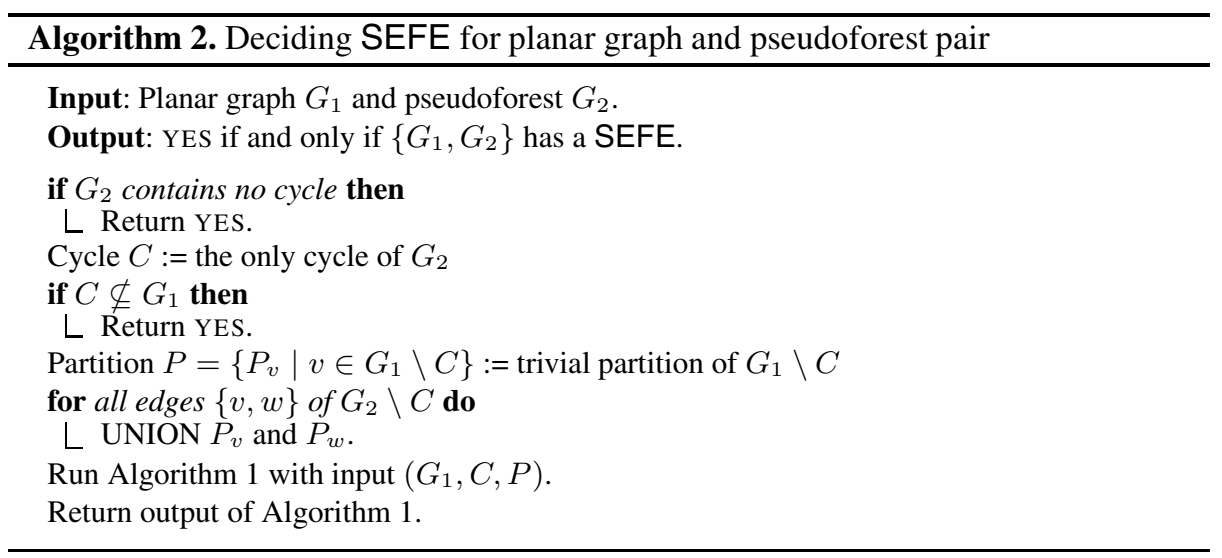

We use the previously discussed results to create an efficient algorithm deciding the problem mentioned in the beginning of this section.

Theorem 5. Algorithm 2 works correctly, i.e., it returns YES if and only if $\left\{G_{1}, G_{2}\right\}$ has a SEFE. Moreover, it has a linear runtime.

Proof. Assume first that the algorithm returns YES, which is by one of three statements. The first returns YES if $G_{2}$ contains no cycle. But then Theorem 2 states that $\left\{G_{1}, G_{2}\right\}$ has a SEFE. The second statement returns YES if cycle $C$ is not completely part of $G_{1}$. Theorem 3 guarantees that $G_{1}$ and $G_{2}$ have a SEFE in this situation. The last instruction is that the run of Algorithm 1 returns YES. Algorithm 1 checks whether graph $G_{1}$ can be embedded in the plane such that all partition sets of $P$ lie completely inside or outside $C$. By the construction of $P$, this is equivalent to saying that both endpoints of every edge of $G_{2} \backslash C$ lie both inside or both outside $C$. Then Theorem 4 yields a SEFE of $G_{1}$ and $G_{2}$.

Assume next, that the algorithm returns NO, which implies Algorithm 1 returned NO. Hence, $G_{1}$ has no planar drawing with the property of Theorem 4, which implies that $G_{1}$ and $G_{2}$ are without a SEFE.

The proposed runtime $O(|V|)$ follows directly from the complexity analysis of Algorithm 1.

\section{A Planar Graph, a Path, and a Cyclic Edge Order}

In this section, we consider two embedding problems with requirements on the cyclic order of some of the edges around a vertex $x$ or two vertices $x$ and $y$ that can be used to decide some special SEFE problems in Section 6 .

In the first problem, $x$ and $y$ are two distinct vertices connected by a path $p$. Let $e_{p}$ and $e_{p}^{\prime}$ be the first and last edges on $p$ incident to $x$ and $y$, respectively, where $\left\{e_{a}, e_{b}\right\}$ and $\left\{e_{a}^{\prime}, e_{b}^{\prime}\right\}$ are distinct edges also incident to $x$ and $y$. We want to ensure that the order of these edges around $x$ and $y$ (amongst other possible incident edges) in a combinatorial embedding $\Gamma$ of $G$ is consistent with an embedding of a graph in which $x$ and $y$ are 
connected by the three edge-disjoint paths $p, p_{a}=e_{a}, \ldots, e_{a}^{\prime}$, and $p_{b}=e_{b}, \ldots, e_{b}^{\prime}$. This implies that either the cyclic order around $x$ is $e_{p}, e_{a}, e_{b}$ and around $y$ is $e_{p}^{\prime}, e_{b}^{\prime}, e_{a}^{\prime}$ or both orders are reversed. It suffices to test only one possibility, since we can generate a combinatorial embedding with the reversed orders simply by mirroring the embedding.

Let $E_{x}=\left\{e_{p}, e_{a}, e_{b}\right\}$ and $E_{y}=\left\{e_{p}^{\prime}, e_{a}^{\prime}, e_{b}^{\prime}\right\}$. We observe that-if not all edges in $E_{x} \cup E_{y}$ are in the same block-such a required combinatorial embedding always exists; in this case, $x$ or $y$ is a cut vertex. We can insert the embedding of one block $B^{\prime}$ into a face of an embedding of the other block $B$ (mirroring the embedding of $B^{\prime}$ if necessary) so that the requirements on the embedding are met. On the other hand, if all the edges in $E_{x} \cup E_{y}$ are contained in a single block $B$, it is sufficient to test a few simple conditions in the SPQR-tree $\mathcal{T}$ of $B$. The necessary and sufficient conditions are given in the lemma below.

Lemma 1. G has a combinatorial embedding $\Gamma$ such that the cyclic order induced by $\Gamma$ on $E_{x}$ is $e_{p}, e_{a}, e_{b}$ and the cyclic order induced on $E_{y}$ is $e_{p}^{\prime}, e_{b}^{\prime}, e_{a}^{\prime}$ if and only if

1. there is no block $B$ of $G$ containing all edges in $E_{x} \cup E_{y}$; or

2. there is a block $B$ containing $E_{x} \cup E_{y}$, and its $S P Q R$-tree $\mathcal{T}$ has neither

(a) a P-node whose skeleton contains three distinct edges $e_{1}, e_{2}, e_{3}$ such that $e_{p}$ and $e_{p}^{\prime}$ are contained in the expansion graph of $e_{1}, e_{a}$ and $e_{b}^{\prime}$ in the expansion graph of $e_{2}$, and $e_{b}$ and $e_{a}^{\prime}$ in the expansion graph of $e_{3}$; nor

(b) an R-node whose skeleton has a combinatorial embedding such that $e_{p}, e_{a}$, $e_{b}$ are in the expansion graphs of three distinct skeleton edges $\tilde{e}_{p}, \tilde{e}_{a}, \tilde{e}_{b}$ in this cyclic order, and $e_{p}^{\prime}, e_{a}^{\prime}, e_{b}^{\prime}$ are in the expansion graphs of three distinct skeleton edges $\tilde{e}_{p}^{\prime}, \tilde{e}_{a}^{\prime}, \tilde{e}_{b}^{\prime}$ in this cyclic order.

These conditions can be checked in linear time, since constructing an SQPR-tree and determining for each edge $e \in E_{x} \cup E_{y}$ in the expansion graphs of which skeleton edges it is contained, requires only linear time, and there are only two combinatorial embeddings of each R-node's skeleton.

In the second embedding problem, we consider a planar graph $G$ with a vertex $x$ and four distinct edges $e_{a}, e_{a}^{\prime}, e_{b}, e_{b}^{\prime}$ incident to $x$. We want to decide if there exists an embedding $\Gamma$ of $G$ that induces a cyclic order on these four edges in which $e_{a}$ and $e_{a}^{\prime}$ (and thus also $e_{b}$ and $e_{b}^{\prime}$ ) are consecutive. The motivation for this problem is similar as for the first problem, where $p$ is an empty path and thus $x$ and $y$ are identical. In this case, deciding if a feasible combinatorial embedding of $G$ exists is even easier. We only need to consider only R-node skeletons containing $x$ in which $x$ is incident to at least four skeleton edges. This gives the following lemma whose conditions can be verified in linear time:

Lemma 2. G has a combinatorial embedding $\Gamma$ such that the cyclic order induced by $\Gamma$ on $E_{x}=\left\{e_{a}, e_{a}^{\prime}, e_{b}, e_{b}^{\prime}\right\}$ is such that $e_{a}$ and $e_{a}^{\prime}$ are consecutive, if and only if either

1. no block of $G$ contains all edges in $E_{x}$; or

2. there is a block B containing all edges in $E_{x}$, and its SPQR-tree contains no R-node whose skeleton $S$ contains $x$ and the edges in $E_{x}$ are in the expansion graphs offour distinct skeleton edges $\tilde{E}_{x}=\tilde{e}_{a}, \tilde{e}_{a}^{\prime}, \tilde{e}_{b}, \tilde{e}_{b}^{\prime}$ such that there exists a combinatorial embedding of $S$ that induces a cyclic order on the edges in $\tilde{E}_{x}$ in which $\tilde{e}_{a}$ and $\tilde{e}_{a}^{\prime}$ are not consecutive. 


\section{Two Planar Graphs with Restrictions and a Decision}

We now consider how this approach of using SPQR-trees might be extended to address more general decision problems for deciding whether a pair of graphs has a SEFE. We examine pairs of planar graphs $G_{1}$ and $G_{2}$ where we restrict both the number and the arrangement of cycles in $G_{2}$ and in $G_{1} \cap G_{2}$.

$\boldsymbol{G}_{\mathbf{1}} \cap \boldsymbol{G}_{\mathbf{2}}$ is a forest: We start with a more general version of Theorem 3 where we have a larger number of cycles in $G_{2}$ but still the intersection is a forest.

Theorem 6. Let $G_{1}=\left(V, E_{1}\right)$ be a planar graph and $G_{2}=\left(V, E_{2}\right)$ be a planar graph where all cycles $C_{i} \subseteq G_{2}, i \in[1 . . k]$, are pairwise disjoint. If no $C_{i}$ is contained in $G_{1} \cap G_{2}$, then the pair $\left\{G_{1}, G_{2}\right\}$ has a SEFE.

Proof (sketch). We adapt the proof of Theorem 3 . When drawing $G_{2}$, remove one edge $e_{i}$ from each $C_{i} \backslash G_{1}$ and draw the rest of $G_{2}$, which is a forest. Then insert one edge $e_{i}$ after another in the same way as done with edge $e$ in the proof of Theorem 3 As all cycles are disjoint and no further cycles exist, this method can be applied without introducing any crossings in the drawing of $G_{2}$.

Next, we discuss the case where $G_{2}$ contains exactly two cycles that either touch in exactly one point or share a common path. With the ideas developed in Section 5 we can handle this situation efficiently.

Theorem 7. The SEFE decision problem for two planar graphs $G_{1}$ and $G_{2}$ where $G_{2}$ contains exactly two cycles and $G_{1} \cap G_{2}$ is a forest can be decided in linear time.

Proof (sketch). Let $C_{1}$ and $C_{2}$ be the two cycles of $G_{2}$. If $C_{1} \cap C_{2}=\emptyset$, the case is trivial as given by Theorem 6 As $G_{2}$ contains no more cycles, $C_{1} \cap C_{2}$ is a path $p$ with endpoints $x$ and $y$; see Fig. 3 . A planar embedding of $G_{1}$ can force the outgoing edges of $x$ and $y$ to have a specific order leading to the situation in Fig. 3 b) in $G_{2}$ that prevents a SEFE of $G_{1}$ and $G_{2}$. However, if $G_{1}$ has an embedding that allows the right cyclic order for both $x$ and $y$ as in Fig. 3(a), then a SEFE can be achieved. All other edges of $G_{2}$ can be drawn without introducing crossings as in the proof of Theorem 4 Lemma 1 gives a linear time check to determine whether $G_{1}$ has an embedding such

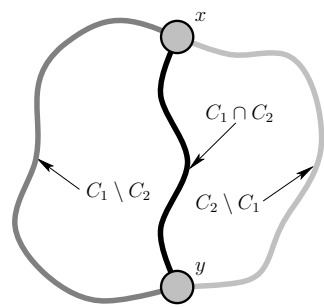

(a)

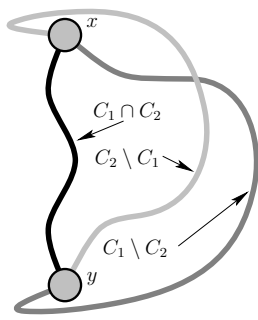

(b)

Fig. 3. The two cycles $C_{1}$ and $C_{2}$ drawn without and with crossings. The respective clockwise ordering of the edges incident to $x$ and $y$ differ. 


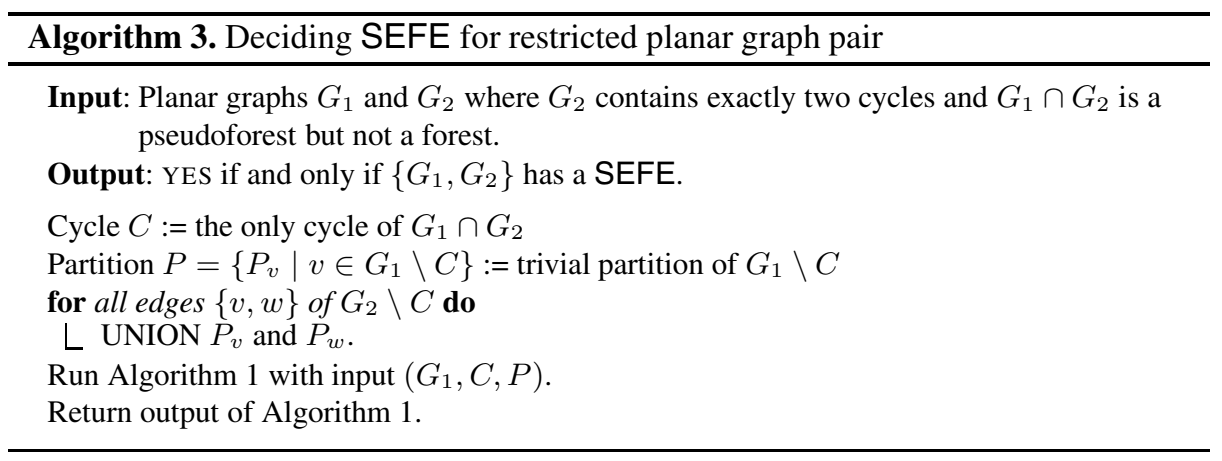

that the cyclic order for the three outgoing edges corresponds to the paths shown in Fig. 3. Lemma 2 handles the degenerate case for $x=y$, also determinable in linear time.

$\boldsymbol{G}_{\mathbf{1}} \cap \boldsymbol{G}_{\mathbf{2}}$ is a pseudoforest: Assume now that both $G_{1}$ and $G_{2}$ are planar graphs in which $G_{2}$ contains exactly two cycles $C_{1}$ and $C_{2}$ of which only one, say $C_{1}$, is contained in $G_{1} \cap G_{2}$. When removing one edge of $C_{2} \backslash G_{1}$ we are in the situation described in Section 4. This correlation allows us to construct a new decision algorithm based on Algorithm 2, We start by generalizing Theorem 4, which we use as the key ingredient to Algorithm 3

Theorem 8. Let $G_{1}=\left(V, E_{1}\right)$ be a planar graph and $G_{2}=\left(V, E_{2}\right)$ be a planar graph with exactly two cycles $C_{1}$ and $C_{2}$ where $C_{1} \subseteq G_{1} \cap G_{2}$ and $C_{2} \not G_{1} \cap G_{2}$. $G_{1}$ and $G_{2}$ have a SEFE if and only if there exists a planar drawing of $G_{1}$ such that for all edges $e=\{v, w\} \in G_{2} \backslash G_{1}$ either both $v$ and $w$ lie inside or both lie outside of $C_{1}$.

Theorem 8 can be proved by using Theorem 4 to determine whether $\left\{G_{1}, G_{2} \backslash\{e\}\right\}$ has a SEFE. In an SEFE of this smaller pair, edge $e=\{v, w\}$ can be inserted if and only if both endpoints $v$ and $w$ lie on the same side of $C_{1}$.

It is easy to see that Algorithm 3 works correctly. We can imitate the proof of correctness of Algorithm 2 (see Theorem 5 ) where this time Theorem 8 plays the role of Theorem 4 .

\section{Concluding Remarks and Future Applications}

We have shown how to use SPQR-trees in the context of simultaneous embedding with fixed edges by presenting several new decision algorithms for some classes of graph pairs. Clearly, much future works remains, but overall this approach of using SPQRtrees seems promising in potentially yielding a polynomial-time decision algorithm for deciding whether two graphs have a SEFE, if one exists. 


\section{References}

1. de Fraysseix, H., Pach, J., Pollack, R.: How to draw a planar graph on a grid. Combinatorica 10(1), 41-51 (1990)

2. Di Battista, G., Didimo, W., Patrignani, M., Pizzonia, M.: Drawing database schemas. Software: Practice and Experience 32(11), 1065-1098 (2002)

3. Di Battista, G., Tamassia, R.: On-line planarity testing. SIAM Journal on Computing 25(5), 956-997 (1996)

4. Di Giacomo, E., Liotta, G.: A note on simultaneous embedding of planar graphs. In: EWCG 2005, pp. 207-210 (2005)

5. Dornheim, C.: Planar graphs with topological constraints. Journal on Graph Algorithms and Applications 6(1), 27-66 (2002)

6. Eiglsperger, M., Fößmeier, U., Kaufmann, M.: Orthogonal graph drawing with constraints. In: Proc. SODA 2000, pp. 3-11 (2000)

7. Erten, C., Kobourov, S.G.: Simultaneous embedding of planar graphs with few bends. In: Pach, J. (ed.) GD 2004. LNCS, vol. 3383, pp. 195-205. Springer, Heidelberg (2005)

8. Estrella-Balderrama, A., Gassner, E., Jünger, M., Percan, M., Schaefer, M., Schulz, M.: Simultaneous geometric graph embeddings. In: Hong, S.-H., Nishizeki, T., Quan, W. (eds.) GD 2007. LNCS, vol. 4875, pp. 280-290. Springer, Heidelberg (2008)

9. Fowler, J.J., Jünger, M., Kobourov, S.G., Schulz, M.: Characterizations of restricted pairs of planar graphs allowing simultaneous embedding with fixed edges. In: WG 2008 (to appear)

10. Frati, F.: Embedding graphs simultaneously with fixed edges. In: Kaufmann, M., Wagner, D. (eds.) GD 2006. LNCS, vol. 4372, pp. 108-113. Springer, Heidelberg (2007)

11. Gassner, E., Jünger, M., Percan, M., Schaefer, M., Schulz, M.: Simultaneous graph embeddings with fixed edges. In: Fomin, F.V. (ed.) WG 2006. LNCS, vol. 4271, pp. 325-335. Springer, Heidelberg (2006)

12. Gutwenger, C., Klein, K., Mutzel, P.: Planarity testing and optimal edge insertion with embedding constraints. In: Kaufmann, M., Wagner, D. (eds.) GD 2006. LNCS, vol. 4372, pp. 126-137. Springer, Heidelberg (2007)

13. Hershberger, J., Suri, S.: An optimal algorithm for Euclidean shortest paths in the plane. SIAM Journal on Computing 28(6), 2215-2256 (1999)

14. Veblen, O.: Theory on plane curves in non-metrical analysis situs. Transactions of the American Mathematical Society 6, 83-98 (1905) 